\title{
ABDOMEN HOSTIL: COMPLICACIÓN QUIRÚRGICA ULTERIOR A 9 INTERVENCIONES
}

\author{
HOSTILE ABDOMEN: SURGICAL COMPLICATION AFTER 9 \\ INTERVENTIONS \\ Dra. Ana María Caballero Arévalo ${ }^{1}$, Dra. Miroslava Daza Caballero ${ }^{2}$, Dr. Carlos Alberto Lara \\ Pérez ${ }^{3}$
}

\section{RESUMEN}

\begin{abstract}
El abdomen hostil es una severa complicación, resultado de múltiples intervenciones quirúrgicas de emergencia realizadas para controlar procesos sépticos abdominales, fugas anastomóticas, complicaciones postoperatorias, y evitar un síndrome compartimental. Estas secuencias quirúrgicas llevan a la creación de ostomías, retracción aponeurótica y formación de adherencias fibrosas intraperitoneales; creando un abdomen de difícil abordaje al momento de la reconstrucción y cierre abdominal, una vez superada la fase aguda. Se presenta el caso clínico de un paciente masculino de 24 años con antecedente de nueve cirugías a raíz de una apendicitis complicada, que lo hace portador de ileostomía, hernia insicional gigante y un abdomen congelado. Acude para la restitución de tránsito intestinal y reparación del defecto herniario, representando un reto quirúrgico de manejo multidisciplinario y un impacto psicológico como económico para el paciente donde las decisiones se basan en los hallazgos intraoperatorios.
\end{abstract}

\section{ABSTRACT}

The hostile abdomen is a severe complication, resulting of multiple emergency surgical interventions, performed to control septic abdominal processes, anastomotic leakage, and postoperative complications and avoid a compartment syndrome. This sequence of surgeries lead to the creation of ostomies, aponeurotic retraction and the formation of intraperitoneal fibrous adhesions; creating a difficult abdomen to approach at the moment of reconstruction and abdominal closure once the acute phase is over. The clinical case of a 24 year-old male patient is presented, with a history of nine surgeries due to a complicated appendicitis, carrying an ileostomy, giant insicional hernia and a frozen abdomen. He came for the restitution of intestinal transit and repair the hernia defect; representing surgical challenge of a multidisciplinary management with high psychological as economic impact for the patient, where decisions are based on intraoperative findings.

\section{INTRODUCCIÓN}

Se define el abdomen hostil como una situaSción donde la cavidad abdominal está abierta, con bordes retraídos, cicatrizada y compactada, en un solo bloque de tejido fibroso que no permite su adecuada disección o separación'.

Al ser consecuencia de múltiples intervenciones, la finalidad es poder realizar un adecuado control de emergencia de patologías que amenazan la vida como peritonitis secundaria, sangrado activo ${ }^{2}$, pancreatitis grave, fuga anastomótica o la utilización de técnicas quirúrgicas de control de daños, abdomen abierto para el manejo del síndrome compartimental, sepsis abdominal, Crohn o carcinomatosis peritoneal'.
Cualquier injuria peritoneal activa la cascada inflamatoria, la angiogénesis, la actividad fibroblástica peritoneal y la condensación de fibrina y colágeno ${ }^{3}$, en consecuencia, se forman bandas fibrosas entre órganos y tejidos, siendo más frecuente entre asas intestinales y la pared abdominal. Ello representa un problema ante una siguiente laparotomía o laparoscopía por la realización de extensas adhesiolisis que impiden una adecuada exposición, teniendo como principal complicación la enterotomía inadvertida con una incidencia del $20 \%$, los tiempos quirúrgicos se prolongan, hay pérdida sanguínea, sepsis y adherencias, entre otras complicaciones ${ }^{2,3}$.

1 Dra. Ana María Caballero Arévalo Cirujano General Jefe del Departamento de Coloproctologia de la Caja Nacional De Salud Hospital Obrero Numero 2

${ }^{2}$ Dra. Miroslava Daza Caballero Medico Genera

${ }^{3}$ Dr. Carlos Alberto Lara Pérez Residente de Cirugía de la Caja Nacional De Salud Hospital Obrero Numero 2

Correspondencia a:

Miroslava Daza Caballero mirosch52@hotmail.com Telf. y celular: 79764596

Palabras clave: Adherencias, abdomen, peritonitis, sepsis.

Keywords: Adhesions, abdomen, peritonitis, sepsis

Recibido para publicación 24 de octubre 2017 Aceptado para publicación: 07 de Julio de 2018

Citar como:

Rev Cient Cienc Med 2018; 21 (1):107-112 
El antecedente de cirugía abdominopélvica condiciona en un periodo de 5-10 años la presencia de adherencias, asociado a elevado costo en relación a reingresos hospitalarios $^{4}$; alrededor de 67 millones en el Reino Unido se deben a dolor abdominal crónico, obstrucción intestinal e infertilidad. Además de representar dificultad, no solo en la exposición de la cavidad sino, extendiendo el tiempo quirúrgico promedio en liberar estas adherencias, que fluctúa entre 1 a 240 minutos, se prolonga el tiempo de anestesia así como la estancia hospitalaria ${ }^{2}$. A pesar de la importancia de esta entidad solo el $14,4 \%$ de los médicos informan sobre la formación de adherencias como complicación y solo el $10 \%$ lo registraron en el consentimiento informado 4 . Por el impacto médico quirúrgico mencionado, asociado a una elevada morbimortalidad, la dificultad de su abordaje terapéutico', y la necesidad de conocer la complejidad de los sucesos que conllevan a su instauración, a continuación se presenta un caso de abdomen hostil y una revisión bibliográfica.

\section{PRESENTACIÓN DEL CASO}

Paciente de sexo masculino de 24 años de edad de profesión maestro, procedente de la provincia Cliza - Cochabamba, presenta cuadro clínico de un año y seis meses de evolución portador de ileostomía y hernia insicional gigante media supra e infraumbilical, acude al módulo de coloproctología del departamento de cirugía de la Caja Nacional de Salud Hospital Obrero $\mathrm{N}^{\circ} 2$ para ser evaluado ante una probable restitución de continuidad intestinal.

Al examen físico consciente, orientado, en buen estado general. Mucosas húmedas y rosadas. Signos vitales: frecuencia respiratoria $18 \mathrm{rpm}$, frecuencia cardiaca $70 \mathrm{lpm}$, presión arterial $120 / 80 \mathrm{mmHg}$, temperatura $36^{\circ} \mathrm{C}$.

Cardiopulmonar clínicamente normal, en abdomen se evidencia cicatriz media supra e infraumbilical con pérdida importante de pared, por donde protruye masa de aproximadamente $15 \mathrm{~cm}$ de longitud por $10 \mathrm{~cm}$ de diámetro; en fosa iliaca derecha se evidencia ileostomía funcional con débito intestinal (ver figura 1). Ruidos hidroaéreos (+) normoactivos, a la palpación superficial pérdida de firmeza abdominal, piel laxa, blando depresible, no doloroso a la palpación profunda ni superficial. Resto del examen sin particularidades

Paciente cuenta con el antecedente de haber sido intervenido quirúrgicamente en múltiples oportunidades cuya evolución y cronología se suscitaron en el siguiente orden.

Diagnosticado de apendicitis complicada un año atrás, a raíz de cuadro agravado por automedicación con múltiples analgésicos, motivo por el que se decide la realización de cirugía de urgencia.

Primera intervención: Tuvieron como hallazgos, un absceso apendicular retrocolónico, necrosis de cara posterior de ciego no logrando evidenciar apéndice cecal. Realizan- do lavado de cavidad peritoneal, hemicolectomía derecha, íleo-transverso anastomosis termino lateral y laparostomía contenida. Se realizó una segunda intervención para revisión de cavidad y una Tercera para laparorrafia y drenaje. Pasó al servicio de Unidad de Cuidados Intermedios (UCIM).

El paciente durante las 48 horas post operatorias cursa con evolución tórpida, presentando una fistula enterocutánea a través de herida quirúrgica, por lo que se decide, una Cuarta intervención: Laparotomía, donde se evidenció contenido fecaloideo en espacio parietocólico derecho, necrosis y dehiscencia de la anastomosis; además de múltiples adherencias inter asas de intestino delgado. Se procede a lavado de la cavidad, resección de zona anastomótica, con rafia en tres planos de muñón del transverso (puntos separados), aproximadamente a $7 \mathrm{~cm}$ del muñón se realiza nueva íleotransverso anastomosis termino lateral, drenaje y laparostomía contenida.

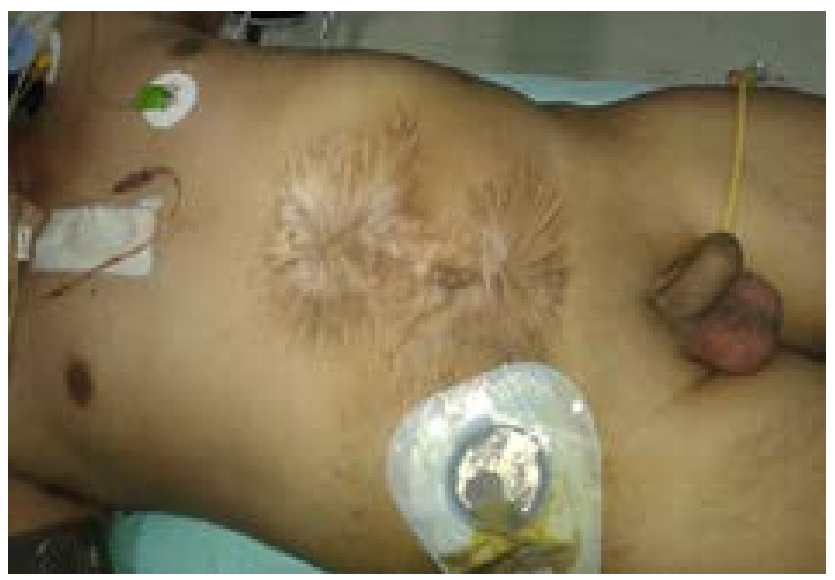

FIGURA 1. Paciente portador de abdomen hostil, hernia insicional supra e infra umbilical, portador ileostomía, previa a intervención quirúrgica

Quinta intervención: Se realiza a los dos días de la cuarta intervención para revisión, toma de muestra para cultivo y antibiograma y lavado de cavidad; en esta oportunidad se pudo evidenciar presencia de fibrina, adherencias laxas inter asas, anastomosis en buenas condiciones, terminando nuevamente en laparostomía contenida para una sexta intervención en la que se realizó laparorrafia y drenaje.

El paciente pasa a Unidad de Cuidados Intensivos (UCI) presentando durante las 72 horas postoperatorias alzas térmicas, abundante debito por sonda nasogástrica, dolor abdominal difuso, contractura muscular abdominal, salida de secreción fecaloidea y maloliente por drenaje en flanco derecho, la biometría hemática indica leucocitosis 19 000, neutrófilos $86 \%$, se reportan cultivos de drenaje compatible con Pseudomona aeruginosa. 
Se decide una Séptima intervención con el diagnóstico de peritonitis, evidenciando fístula de muñón de colon transverso, asas de intestino delgado edematizadas, múltiples adherencias inter asas firmes y peritoneo, absceso en cara posterior de colon transverso, realizándose lavado, drenaje y resección de anastomosis, cierre de cabo proximal en tres planos, ileostomía terminal y laparotomía contenida.

Octava intervención: se evidenció aponeurosis retraída, múltiples adherencias inter asas firmes, abdomen congelado, perforación en yeyuno de aproximadamente $5 \mathrm{~mm}$, el manejo fue yeyunostomía con sonda Foley tipo Stam, se deja al paciente en laparotomía contenida.

Novena intervención: nueva revisión dos días posteriores, donde se decide rafia de pared abdominal.

El paciente presenta una evolución con dehiscencia de sutura de piel, salida de secreción seropurulenta la cual se lleva a estudio de antibiograma y es compatible con Staphylococcus Aureus, la misma que se resuelve con curaciones diarias, antibiótico terapia y cierre por segunda intención de piel. Se reporta cierre espontáneo de fístula enterocutánea yeyunal puntiforme de escaso debito tres meses posteriores.

Interconsultas y resultados recientes

Basados en los antecedentes se realiza un estudio de colon por enema, evidenciándose el paso libre de contraste hasta colon transverso, además de tomografía axial computarizada que muestra diátesis de rectos supra e infraumbilical, distancia de defecto de pared muscular $25 \mathrm{~cm}$, hernia insicional gigante. Ecografía de pared reporta región supraumbilical izquierda, asas intestinales separadas de la piel por delgada capa de tejido celular subcutáneo compatible con una eventración (ver figura 2).

Se realiza interconsulta por nutrición para mejorar la ganancia ponderal pre quirúrgica y con el módulo de tratamiento quirúrgico de pared abdominal, para la evaluación del defecto herniario y la importante retracción aponeurótica, quienes proponen la reparación mediante la técnica de separación de componentes, y utilización de malla.

Tratamiento aplicado

Internación para preparación colónica a base de limonada laxante 1 litro por día a tolerancia, preparación mecánica con enema evacuante por recto, solución Ringer normal 1 000 cc por vía endovenosa, metronidazol 500 mg VO cada 8 horas, omeprazol $50 \mathrm{mg}$ EV cada 12 horas.

Diagnóstico preoperatorio: Portador de ileostomía, hernia insicional gigante

Diagnóstico postoperatorio: Abdomen Hostil

Se ingresa a procedimiento quirúrgico evidenciando cicatriz cutánea retráctil irregular, anillo herniario de más o menos $20 \mathrm{~cm} \times 15 \mathrm{~cm}$, saco herniario que contiene asas de intestino delgado, retracción aponeurótica de aproximadamente $15 \mathrm{~cm}$. Múltiples adherencias inter asas y apelotonamiento de intestino delgado con adherencias a pared abdominal anterior (abdomen congelado), (ver figura 3).
Ileostomía terminal en asa ( $\mathrm{J}$ ) permeable, Íleon adherido a pared abdominal anterior en dos segmentos y hernia paraestomal.

Se liberan adherencias de intestino a pared abdominal, manejo en bloque de asas de intestino delgado y exteriorización con liberación de adherencias inter asas, (ver imagen 4). Luego, resección de íleon proximal por pérdida de serosa, restitución del tránsito intestinal íleo-transverso, anastomosis termino lateral, hernioplastía insicional con malla de polipropileno con técnica de separación de componentes y drenaje.

Paciente es trasladado al servicio de unidad de cuidados intensivos ( $\mathrm{UCl}$ ) para su monitorización, instauración de catéter central y alimentación parenteral, control hidroelec-
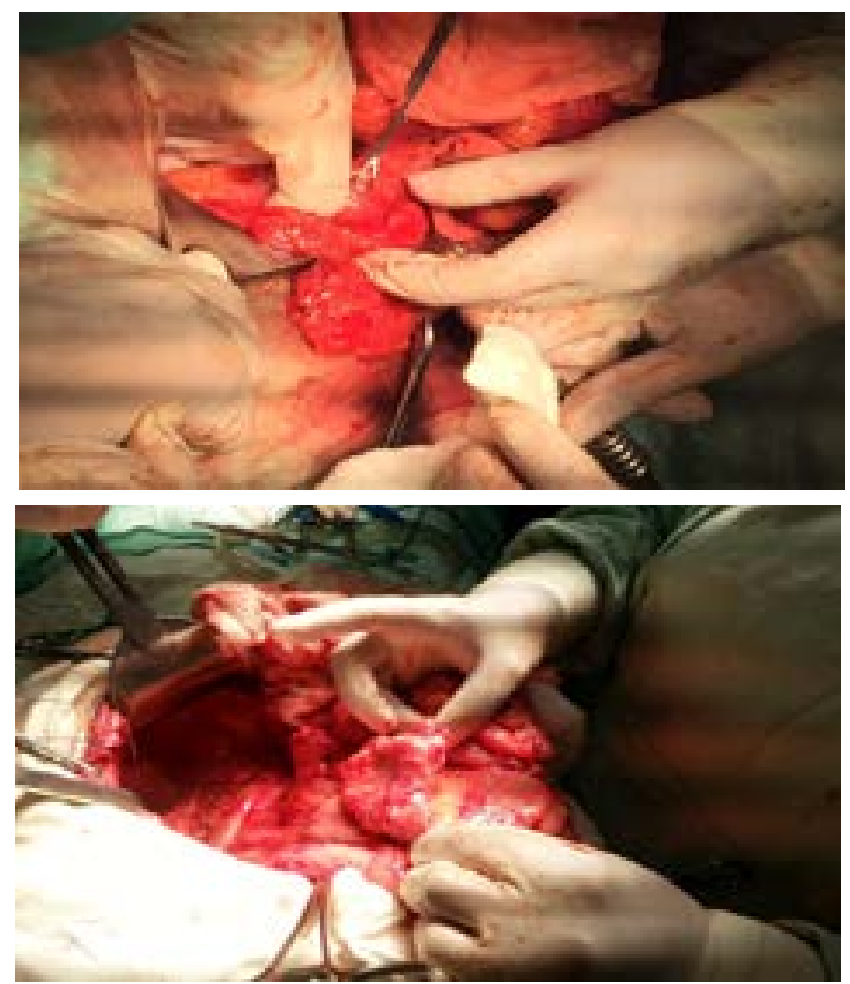

FIGURA 2. Obsérvese la importante formación adherencial de la cavidad abdominal ,figura a) abdomen congelado figura b) liberación de adherencias firmes de asas intestinales a pared abdominal

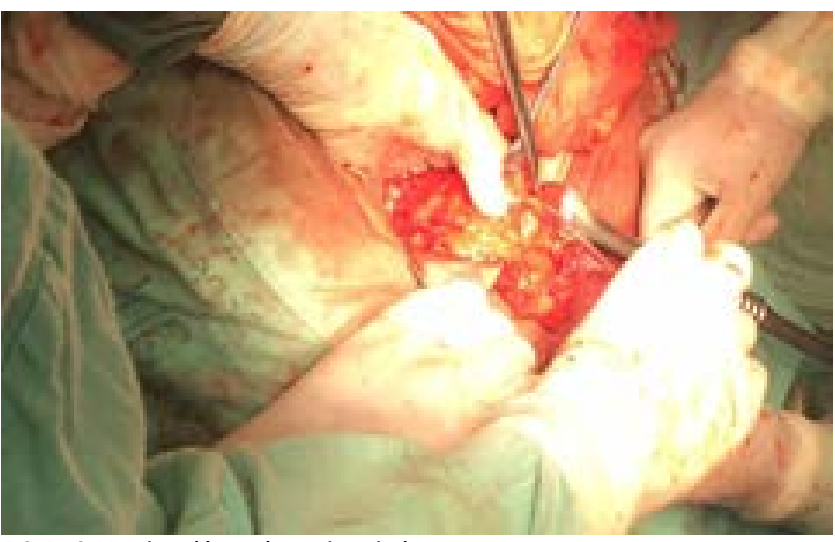

FIGURA 3. Manejo en bloque de asas intestinales 
trolítico estricto e inicio de antibioticoterapia, con posterior manejo en salas generales hasta tolerancia de la vía oral, con evolución favorable y alta hospitalaria diez días después de su intervención quirúrgica. Se indica seguimiento por nutrición y consulta externa cada quince días por dos meses y cada seis meses por un año.

\section{DISCUSIÓN}

Al presentarse una apendicitis complicada de larga evolución con posterior formación de un absceso peri apendicular severo, el manejo de la base apendicular se dificulta como consecuencia del proceso inflamatorio, necrosis de pared cecal y el posible compromiso de órganos vecinos; la apendicetomía por si sola es insuficiente, siendo en ocasiones necesaria la resección ileocecal o hemicolectomía derecha, para prevenir complicaciones sépticas como fístula intestinal, peritonitis, abscesos intrabdominales y obstrucción intestinal aguda ${ }^{5}$.

La anastomosis primaria posterior a una colectomía derecha de emergencia es considerada como estándar ${ }^{6,7}$, sin embargo, Hahnloser et al. en un estudio comparativo, demostró que ante mayor riesgo pre quirúrgico debido a comorbilidades, sepsis y estado hemodinámico adverso, mayor porcentaje de fuga anastomótica en relación a unión primaria y mayor porcentaje de ileostomía permanente, siendo recomendable en estos pacientes una ileostomía temporal o laparostomía?.

Otros factores que influyen en la dehiscencia o posterior instauración de fistula enterocutánea, son el nivel de la anastomosis, asociándose caudalmente a mayor riesgo de fuga en relación a flora bacteriana abundante y menor vascularización, observándose a nivel colorectal $5 \%$ más de fuga, la resección de emergencia, hipoxia y vasoconstricción relacionadas, enfermedad pulmonar, cardiovascular o diabetes, uso de corticoides sistémicos prolongados, excesiva tensión y asimetría entre los cabos de acoplamiento anastomóticos, derivan en diferentes grados de severidad de dehiscencia ${ }^{6}$ el manejo se guía por el gasto a través del dre-

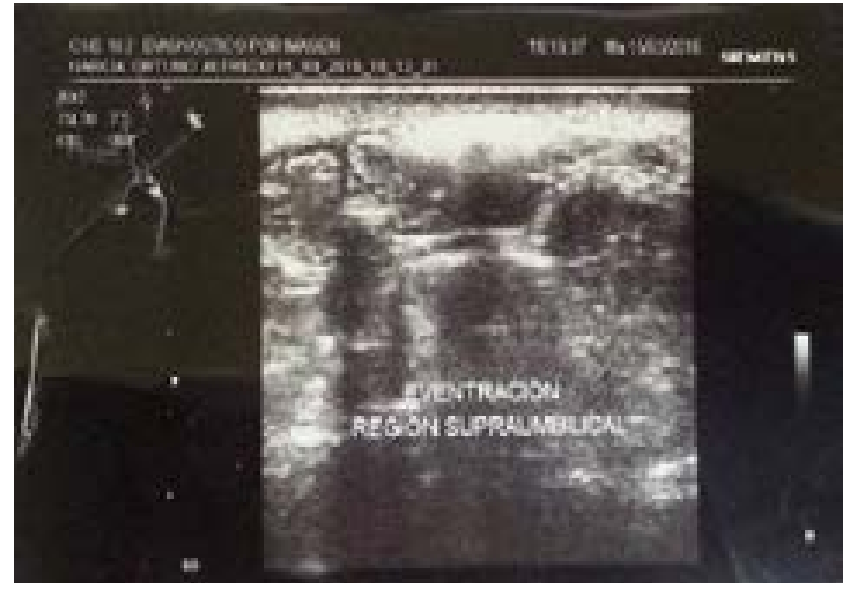

IMAGEN 4. Ultrasonido donde se evidencia la gran retracción de la pared abdominal

naje o la piel, considerado como debito bajo, $<500 \mathrm{ml} / 24 \mathrm{hr}$, las opciones incluyen tratamiento médico y drenaje percutáneo dirigido, sin embargo un debito alto $>500 \mathrm{ml} / 24 \mathrm{hrs}$ acompañado de alteración hemodinámica o signos de sepsis se resuelve a través de una ostomía temporal ${ }^{6,8}$.

En el presente caso la decisión de emergencia fue realizar una anastomosis íleo-transversa primaria, sin embargo las condiciones sépticas llevaron a la dehiscencia y consecuentes intervenciones quirúrgicas que con la intención de controlar el proceso séptico y la finalidad de restaurar la continuidad intestinal como prioridad, llevaron a la formación de adherencias firmes, enterotomías inadvertidas; además de retracción aponeurótica, que derivaron en la instauración de una ileostomía temporal y un abdomen hostil. De acuerdo a la evidencia mencionada podría evitarse al instaurar una ileostomía de forma temprana. Sin embargo, es difícil predecir la frecuencia de esta complicación, por las múltiples variables a considerar, pudiendo presentar inclusive un $30 \%$ de fracaso posterior a la restitución del tránsito intestinal definitiva por la presencia de nuevas fugas anas-

TABLA 1

\begin{tabular}{|c|l|}
\hline \multicolumn{2}{|c|}{ Clasificación de Björck 2009} \\
\hline Grado & Descripcion \\
\hline $1 \mathrm{a}$ & Limpio sin adherencias \\
\hline $1 \mathrm{~b}$ & Contaminado sin adherencias \\
\hline $2 \mathrm{a}$ & Limpio con adherencias fijas \\
\hline $2 \mathrm{~b}$ & Contaminado con adherencias fijas \\
\hline 3 & $\begin{array}{l}\text { Abdomen abierto complicado, con } \\
\text { fistula en formación }\end{array}$ \\
\hline 4 & $\begin{array}{l}\text { Abdomen abierto congelado, adheren- } \\
\text { cias firmes a intestino, imposible de } \\
\text { cerrar, con sin fistula }\end{array}$ \\
\hline
\end{tabular}

tomóticas ${ }^{8}$.

Utilizar el abdomen abierto o laparostomía, como estrategia para el control de la peritonitis secundaria, tienen como principales indicaciones la revisión secundaria de cavidad, evitar la instauración de síndrome abdominal compartimental y mejorar el control de secreciones infecciosas, sin embargo, las consecuencias desfavorables como pérdida hidroelectrolítica, evisceración, y formación de fístulas entero atmosféricas predisponen a mayor infección de cavidad e importantes adherencias que imposibilitan aún más la resolución quirúrgica9 ${ }^{9}$. Tomar en cuenta que a mayor tiempo de permanencia y número de revisiones en un abdomen abierto, mayor dificultad en el cierre aponeurótico ${ }^{10}$. Sin embargo, se describen actualmente múltiples técnicas para el cierre 
temporal del abdomen abierto que reducen estas complicaciones, como el sistema de cierre asistido por vacío "vacuum assisted closure“" (V.A.C) que conectado a un sistema de succión a presión negativa, mostró superiores beneficios en relación a drenaje de secreciones, cierre de pared y menor formación de fístulas con eventual curación y prevención de su formación al instaurarse de manera precoz ${ }^{11,12}$.

La clasificación de Björck del abdomen abierto es una estrategia para determinar el grado de adherencias en la cavidad abdominal y posicionar su complejidad en cuatro grados desde escenarios simples hasta los más complejos para prevenir mayor deterioro y realizar acciones oportunas para su manejo ${ }^{13,14}$ (ver tabla1); comparando las características descritas en el caso, se posiciona dentro de un grado 4 de Björck el cual describe un abdomen abierto congelado con adherencias firmes a intestino, imposible de cerrar sugiriéndose como conducta el devolver la fisiología y la nutrición del paciente a la normalidad, proteger la piel y la fascia, previniendo la sepsis y posponiendo la reconstrucción compleja, generalmente entre 6 y 12 meses $^{14}$.

El manejo del abdomen hostil requiere de un profundo conocimiento de las potenciales complicaciones que pueden acontecer durante las etapas evolutivas mal controladas ${ }^{15}$, tales como falla intestinal, peritonitis, sepsis, formación de abscesos, fístulas enterocutáneas, entero atmosféricas, uso de nutrición parenteral prolongada, síndrome de intestino corto a raíz de múltiples resecciones, oclusión intestinal recurrente y altos costos en la atención'. En este entendido, el manejo de este tipo de pacientes debe ser dirigido por un equipo multidisciplinario en la Unidad de Cuidados Intensivos (UCl) teniendo la experiencia para afrontar este reto quirúrgico, de forma individualizada e íntegra ${ }^{15,16}$. Los pilares del tratamiento ante la presencia de un abdomen hostil se basan en la estabilización hemodinámica oportuna, instauración de terapia antimicrobiana empírica, adecuada nutrición, y control oportuno de la fuente infecciosa utilizando el manejo del abdomen abierto, junto a técnicas de cierre temporal para permitir múltiples revisiones abdominales, reducir el trauma continuo de la pared abdominal y controlar todas las complicaciones mencionadas ${ }^{17}$.

Una vez superada la fase aguda antes de pensar en re intervenir con intención de restaurar lacontinuidad gastrointestinal o corregir los defectos de la pared abdominal es necesario que el paciente se encuentren en óptimas condiciones hemodinámicas, metabólicas, nutricionales y en ausencia de infección ${ }^{16}$ acompañado de un adecuado apoyo y seguimiento por psicología y nutrición además de un diagnóstico imagenológico completo para acercarse con mayor precisión a la sospecha diagnóstica, previo a la cirugía definitiva de las complicaciones del abdomen hostil ${ }^{12}$. Considerar el abordaje cuidadoso periférico al proceso adherencial, aislamiento en bloque de las asas adheridas, evitando sobre todo la lesión intestinal sin dejar de lado la posibilidad de realizar estomas definitivos ${ }^{15,16,17}$. Las decisiones quirúrgicas dependen de los hallazgos intraoperatorios, planteamiento de objetivos que beneficien al paciente, minimización de riesgos y realizadas en manos experimentadas. Sin embargo, por tratarse de una patología de gran complejidad, elevada mortalidad, y la presentación de la misma no reconocida en su momento como abdomen hostil, dificulta ser descrita como tall ${ }^{15}$.

La presencia de un defecto importante en la pared abdominal como resultado esperable, secundario a retracción aponeurótica, debe ser en efecto reparado conjuntamente como paso final, teniendo múltiples opciones; técnica de separación de componentes, prótesis de malla artificial o tejido autólogo con colgajos pediculares o micro vasculares, las cuales deben ser adaptadas individualmente ${ }^{18}$. Por las características del paciente en el caso clínico, se aplicó la técnica de separación de componentes debido a su utilidad en retracciones mayores a 10 centímetros, menor porcentaje de recidiva, y la combinación con tejido autólogo o protésico para evitar la tensión de la pared abdominal ${ }^{16,19}$ observándose resultados satisfactorios.

\section{REFERENCIAS}

1.Prieto M, Guzmán Ó, García-Morales J. Abdomen Hostil. En: asociación Mexicana de cirugía general A.C., Federación Mexicana de colegio de especialistas en cirugía general. Tratado de cirugía general. 3rd ed. Distrito Federal: Editorial El Manual Moderno; 2017.p. 1203-11

2.Van Goor $\mathrm{H}$. Consequences and complications of peritoneal adhesions. Rev . Colorectal Dis [Internet]. 2007 [Acceso 5 de Septiembre 2017]; 9(suppl 2):25-34. Disponible en: http:// onlinelibrary.wiley.com/doi/10.1111/j.1463-1318.2007.01358.x/ abstract

3.Attard J.A., MacLean A.R. Adhesive small bowel obstruction: epidemiology, biology and prevention. Rev Can J Surg [Internet]. 2007 [Acceso 2 de septiembre 2017; 50(4): 291-93. Disponible en : https://www.ncbi.nlm.nih.gov/pubmed/17897517

4.Wilson M. Practicalities and costs of adhesions. Rev Colorectal Dis [Internet]. 2007 [Acceso 6 de Octubre 2017]; 9(Suppl 2): 60-5. Disponible en: https://www.ncbi.nlm.nih.gov/pubmed/17824972
5.Radionov Y. Appendectomy and Resection of the Terminal Ileum with Secondary Severe Necrotic Changes in Acute Perforated Appendicitis. Rev Am J Case Rep [Internet]. 2015 [Acceso 8 Noviembre 2017];16:37-40. Disponible en: https://www. ncbi.nlm.nih.gov/pubmed/25618525

6.Ruggiero R, Sparainga L, Docimo G, Gubitosi A, Agresti M, Procaccini E, et al. Post-operative peritonitis due to anastomotic dehiscence after colonic resection. Multicentric experience, retrospective analysis of risk factors and review of the literature. Rev Ann Ital Chir [Internet]. 2011 [Acceso 8 Noviembre 2017]; Disponible en: https://pdfs.semanticscholar.org/784e/5464f4afb7aal 564cbac239619a6ee94e8ba.pdf

7.Teixeira H, Melloul E, Hahnloser D, Demartines N, Hübner M. Emergency right colectomy: which strategy when primary anastomosis is not feasible?. Rev World J Emerg Surg [Internet].2016 [Acceso 8 Noviembre 2017];11(19):1-6. Disponible en: https://www. ncbi.nlm.nih. gov/pmc/articles/PMC4855428/ 
8.Martínez JL, Luque-de León E, Román R, Juárez MA, Méndez JD. Factores de riesgo y evolución de fístulas enterocutáneas posterior a cierre de ostomías terminales.Rev Cir y Cirj [Internet].2013 [Acceso 11 Diciembre 2017]; 81(5):394-399. Disponible en: http:// www.redalyc.org/articulo.oa? $i d=66228814005$

9.Atema J, Gans S, Boermeester M. Systematic Review and Meta-analysis of the Open Abdomen and Temporary Abdominal Closure Techniques in Non-trauma Patients. Rev World J Surg [Internet].2014 [Acceso 6 de septiembre 2017]; 39(4):912-925. Disponible en: https://link.springer.com/ article/10.1007\%2Fs00268-014-2883-6

10.Coccolini F, Biffl W, Catena F, Ceresoli M, Chiara O, Cimbanassi $\mathrm{S}$, et al. The open abdomen, indications, management and definitive closure. Rev World $\mathrm{J}$ Emerg Surg [Internet ].2015 [Acceso 6 de octubre 2017]; 10(32)1-10. Disponible en: https://doi.org/10.1186/s13017-015-0026-5

11.Quyn A.J, Johnston C, Hall D, Chambers A, Arapova N, Ogston $\mathrm{S}$, et. al. The open abdomen and temporary abdominal closure systems-historical evolution and systematic review. Rev Colorectal Dis [Internet].2012 [Acceso 6 de octoctubre 2017] ;14(8):429-38. Disponible en: http://onlinelibrary.wiley.com/doi/10.1111/j.14631318.2012.03045.x/abstract

12.Ivatury R. Update on Open Abdomen Management: Achievements and Challenges. Rev World J Surg [Internet].2009 [Acceso 20 octubre 2017]; 33(6):1150-53. Disponible en: https:// link.springer.com/article/10.1007\%2Fs00268-009-0005-7

13.Rivera M, Quezada B, Quiñónez M, Almada M, Manejo de estomas complicados $\mathrm{y} / \mathrm{o}$ abdomen hostil con la tecnica de condon de Rivera diez años de experiencia. Rev Cirujano $G$ [Internet].2017 [Acceso 2 de abril de2018];39(2): 82-9. Disponible en: http://www.medigraphic.com/pdfs/cirgen/cg-2017/cg172d.pdf

14.Björck M, Bruhin A, Cheatham M, Hinck D, Kaplan M, Manca G et al. Classification Important Step to Improve Management of Patients with an Open Abdomen. Rev World J Surg [Internet].2009 [Acceso 2 de abril de 2018];33(6):1154-1157. Disponible en: https:// link.springer.com/content/pdf/10.1007\%2Fs00268-009-9996-3.pdf 15.Leppäniemi A.K Editor-in-Chief. The hostile abdomen-A systematic approach to a complex problema. Rev Scand J Surg [Internet].2008 [Acceso 2 de septiembre 2017]; 97: 218-19. Disponible en: http://journals.sagepub.com/doi/ pdf/10.1177/145749690809700301

16.Latifi R. Joseph B. Kulvatunyou N. Wynne J.L. O'Keeffe T. Tang A. et al. Enterocutaneous Fistulas and a Hostile Abdomen: Reoperative Surgical Approaches. Rev World J Surg [Internet].2012 [Acceso 6 de septiembre 2017]; 36(3): 516-23. Disponible en: https://doi.org/10.1007/s00268-011-1306-1

17.Asociación Mexicana de Cirugía General A.C., Colegio de Posgraduados en Cirugía General A.C., Federación Nacional de Colegios y Asociaciones de Especialistas en Cirugía General A.C. Comité de elaboración de guías de práctica clínica México. Abdomen hostil. Guía de práctica clínica [Internet].2014 [Acceso 6 octubre 2017]. Disponible en: https://amcg.org.mx/images/ guiasclinicas/abdomen hostil.pdf18. Leppäniemi A, Tukiainen E. Reconstruction of complex abdominal wall defects. Rev Scand J Surg [Internet].2013 [Acceso 10 septiembre 2017];102(1):149. Disponible en: https://doi.org/10.1177/14574969131020010 419. Cuenca O, Ferreira R, Martínez N. Técnica de separación de componentes con refuerzo de malla de polipropileno para el tratamiento de afecciones parietales complejas. nuestra experiencia. Rev. Cir. Parag [Internet].2015 [Acceso 11 Diciembre 2017];39(1):17-21. Disponible en : http://scielo.iics.una.py/scielo. php? script $=$ sci_arttext\&pid $=S 2307-04202015000100004 \& \operatorname{lng}=e n$ 